\title{
Research on the Construction of Cloud ERP Ecosystem Security Evaluation Index System
}

\author{
Zhujun WANG, Qin SU ${ }^{1}$, Yue FANG \\ School of Management, Xi'an Jiaotong University, Xi'an, China
}

\begin{abstract}
Cloud ERP system realizes the functions of ERP in the way of cloud service, which greatly improves the service level of ERP and reduces the cost for enterprise to use ERP. Cloud ERP system has the characteristics of open ecology, therefore the interactions between users increase, which brings data security risks to the cloud ERP platform. This paper discusses the security of cloud ERP system from the perspective of ecosystem, and puts forward the concept and connotation of cloud ERP ecosystem. The LDA method is used to analyze the literature related to the security of ecosystem and cloud ERP open ecology, based on which seven main themes are summarized. It mainly focuses on system risk assessment, information security management and platform ecosystem construction. Based on LDA analysis results, this paper constructs a cloud ERP ecosystem security evaluation system using expert interviews from three dimensions: developer ecosystem security, user ecosystem security and device-system- user ecosystem security.
\end{abstract}

Keywords. Cloud ERP, ecosystem, text analysis, Cloud ERP ecosystem security evaluation system

\section{Introduction}

Cloud ERP system is the most commonly used cloud computing system in enterprises. Compared with the traditional ERP system, the cloud ERP system is more convenient. It breaks through the geographical restrictions and meets the needs of enterprises to move and work anytime and anywhere. However, due to the particularity of its environment and the architectural characteristics of its products, the security of cloud ERP has become a concern. According to the survey, $80 \%$ of users consider security as the primary indicator for whether to mi grate their business to ERP cloud platform. It can be seen that security has become the bottleneck affecting the promotion of ERP cloud product. There is urgent demand for rational analysis of the mechanism of cloud ERP system security problems, as well as scientific assessment of the security risks of cloud ERP system.

Compared with the traditional ERP system, the cloud ERP system has more information transmission and more interaction between users and the system, which makes the system dynamic and open. By referring to the competitive and cooperative process of population and community in the ecosystem, the interaction among developers,

\footnotetext{
${ }^{1}$ Corresponding Author: Qin SU, School of Management, Xi'an Jiaotong University, Xi'an, China. Email:qinsu@mail.xjtu.edu.cn
} 
systems and users in the cloud ERP system can better reflect the dynamic, ecological and open nature of cloud ERP system.

\section{Literature review}

\subsection{Cloud ERP and security literature review}

Cloud ERP system eliminates the bottleneck of traditional ERP, such as high investment cost, low success rate of implementation, and great difficulty in operation and maintenance However, the interaction of cloud ERP system poses a threat to the user information and data security of the platform[1]. GUPTA et al.[2] and some other researchers pointed out that security risk is the biggest threat to the adoption of cloud ERP for both small and medium-sized enterprises and large enterprises. Erdal Cayircil et al.[3] proposed a cloud adoption risk assessment model, providing automatic analysis of the risks of representative cloud service providers from the aspects of security, trust and guarantee registration of cloud security alliance, so as to help select the cloud service providers with the lowest risk in configuration. Chandramohan.D et al.[4] proposed hybrid authentication technology to protect user data privacy. LEWANDOWSKI et al.[5] pointed that the information-based enterprise has a significant impact on the adoption of SaaS ERP. The adoption of cloud ERP can be realized smoothly only if the enterprise has certain capital, technology and management foundation in informatization. Customers with higher awareness on informatization can make better use of their own advantages and appropriately adopt cloud ERP according to their own needs.

\subsection{Ecosphere and security literature review}

The concept of biosphere in biology has been gradually applied to study various fields. Lu Yibo et al.[6] studied the driving role of universities in open innovation ecosystem. With the concept of ecosystem, Tong Yuqing et al.[7] focused on the core business of ecommerce in the business system of Alibaba Group and the financial business supporting the e-commerce system. They study a business ecosystem that integrates local living, health and recreation. Qian Xiaocong[8] proposed the concept of big data industry ecosystem, and studied and analyzed the composition of the big data industry from the perspective of with the tools of system, community, population and so on, trying to interpret the division and relationship of each part of the industry from the perspective of length and breadth.

\subsection{Cloud service security evaluation index system literature review}

Xue Shan[9] outlined the cloud service business and security management requirements of small and medium-sized financial enterprises, and according to the security risks they are faced with, a cloud service security management framework was built. Then, the cloud service security management evaluation system is formed Xuexiu Chen et al.[10] proposed a complete set of cloud security assessment indicators based on classification and gradation. And a comprehensive evaluation method including positive evaluation 
and feedback evaluation is proposed to verify the rationality and effectiveness of the cloud security evaluation index system.

To sum up, previous studies pointed out the advantages of cloud ERP system and security threats to cloud ERP systems have attracted extensive attention. Current research focuses on the analysis and description of security issues and ignores the impact of cloud ERP ecosystem's ecological openness on system security. This paper proposes the concept of cloud ERP ecosystem from the perspective of open innovation ecosystem, and discusses the relationship between cloud ERP ecological openness characteristics and information security of system. The existing evaluation indicator system in the field of cloud service is oriented towards the whole cloud service. But there is no system for evaluation indicator specified for the security of cloud ERP. Moreover, most of the studies are based on the analysis and improvement of existing evaluation indicators, or subjective extraction of indicators from existing studies and standards. This paper utilizes the method of text analysis to sort out papers related to ecosystem security and openness of cloud ERP ecology, and summarizes specific topics. Based on the concept of cloud ERP ecosystem and the system of evaluation indicator, the security evaluation system of cloud ERP ecosystem is constructed to help improve the information security level of cloud ERP system.

\section{Construction of cloud ERP ecosystem}

Traitler $\mathrm{H}$ et al[11] proposed the concept of open innovation ecosystem to study the change of innovation paradigm; Yu Yao[12] proposed the concept of e-commerce ecosphere and studied the collaborative mechanism of e-commerce ecosphere; Tong Yuqing et al.[13] proposed the concept of business ecosphere to study the collaboration of Internet technology and service terminals in the business system of Alibaba Group; Qian Xiaocong[14] proposed the concept of big data industry ecosystem to analyze the composition of big data ind ustry. This paper draws on the concept of ecosphere in various fields. Based on the concepts of system, community and population in ecology, the concept of cloud ERP ecosystem was further analyzed. Cloud ERP ecosystem is a dynamic and organic system composed of developers, users and software systems with cloud ERP system at its core. Cloud ERP ecosystem contains three levels of meaning: developers constitute the ecosystem, the ecosystem of users and developers, users and cloud ERP system.

\subsection{Developer ecosystem}

The ecosystem composed of developers is mainly oriented towards the service functions of the system. One of the core ideas of cloud computing is to reduce the processing burden on the user end by continuously improving the processing capacity of the cloud. The user end is reduced to a single input-output device, and enjoys the powerful computing capability of the cloud as needed. Cloud ERP represents the integration of the advantages of cloud computing and traditional ERP system. At present, the projects of technical research and exchanges in open source mode have covered various fields such as big data architecture, data query, data processing, workflow coordination, statistical tools, machine learning and cloud computing. System developers jointly constitute the 
development ecosystem to realize the sharing of technology, platform and data, and jointly improve the service functions of the system.

\subsection{User ecosystem}

The ecosystem composed of cloud ERP users is mainly the application object of the system. For an enterprise, the users of the cloud ERP system are distributed in a pyramid structure, as shown in Figure 1. It includes three main groups. First, procurement, production, inventory, sales or finance worker, who is responsible for the data entry system. The second is the data analyst, who monitors and analyzes the data in the system to generate periodic reports for decision-making. On the top is the management level of the enterprise. Through the data retained by the system, the management level identifies risks faced by the enterprise and carries out macro control over the enterprise.

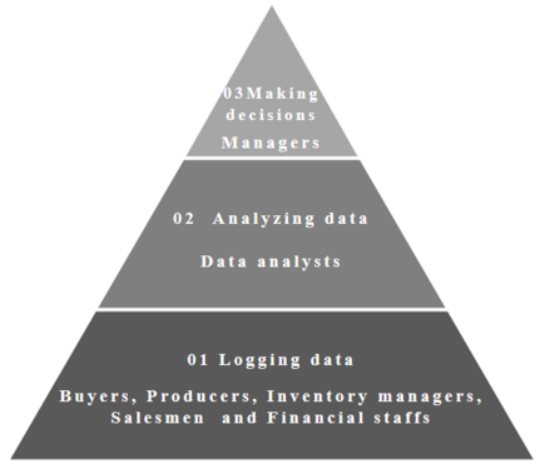

Figure 1. Pyramid structure of user ecosystem within an enterprise.

All system users in the enterprise together constitute the user ecosystem. From the perspective of industry distribution, cloud ERP system is widely used, and users came from many enterprises in the fields of government, energy, telecommunications, manufacturing and medical care, which together constitute the user ecosystem.

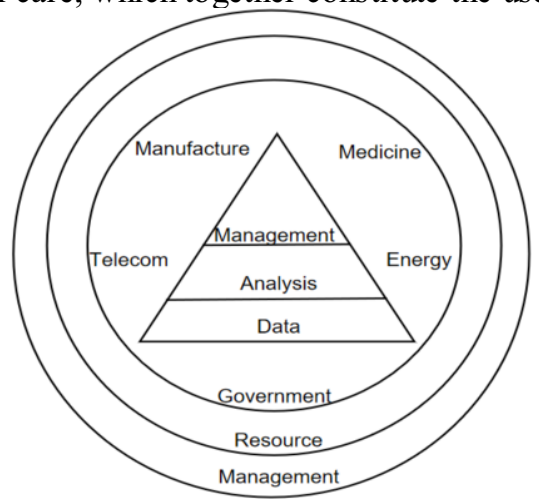

Figure 2. User ecosystem.

\subsection{Developer-system-user ecosystem}

Cloud ERP system developers, users and the cloud ERP system interact with each other and form an organic dynamic ecosystem. Cloud ERP system developers complete the construction of the cloud ERP system and realize system's various service functions; 
Cloud ERP platform is Shared by many users, and business data of enterprises are retained on the system side to achieve data centralization. It optimized the process of enterprise data input, storage and management, improved the system efficiency among various departments of the enterprise, and reduced the labor cost. System developers and many users interweave and influence each other through the cloud ERP system, forming a collaborative ecosystem together, as shown in the figure below.

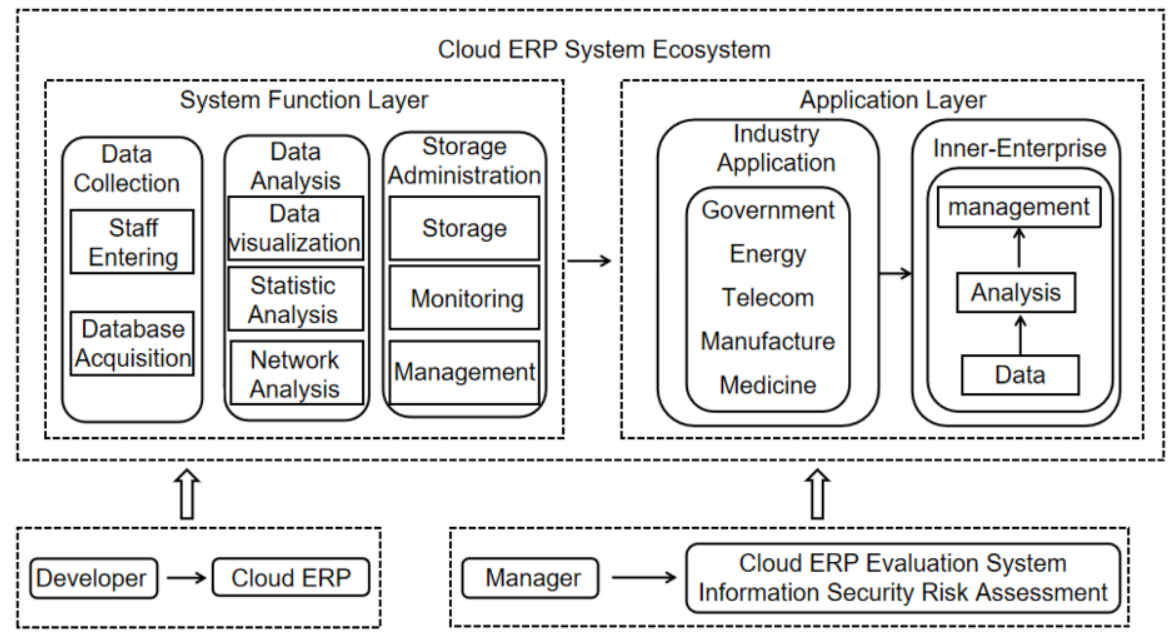

Figure 3. Cloud ERP system ecosystem.

\section{Text analysis based on LDA}

At present, most researches on the evaluation index system of cloud security are based on the analysis and improvement of existing evaluation indexes, or subjective extraction of indexes from existing researches and standards, which make the evaluation index system become less scientific and applied. Therefore, in view of this deficiency, LDA method is adopted in this study to analyze the documents related to the ecological opening characteristics of ecosphere, security and cloud ERP, and summarize the characteristic literature to obtain the research topic[15], which provides the basis for the construction of cloud ERP ecosystem security evaluation system.

\subsection{The topics of ecosphere and security field}

The security of ecosphere is the focus of scholars' attention. LDA is used to analyze the document set related to the ecosystem and ecosystem safety, as shown in Table 1.

Table 1. Most relevant term frequencies for the biosphere and biosphere security.

\begin{tabular}{cccccc}
\hline$\#$ & Term & Frequency & $\#$ & Term & Frequency \\
\hline 1 & Technology & 998 & 16 & Market & 601 \\
2 & Data & 976 & 17 & Organization & 595 \\
3 & Ecosystem & 956 & 18 & Institution & 584 \\
4 & Develop & 903 & 19 & Value & 570 \\
5 & Business & 878 & 20 & Relationship & 554 \\
6 & Innovation & 865 & 21 & Resource & 543 \\
7 & Platform & 843 & 22 & Process & 453 \\
8 & Service & 801 & 23 & Change & 403 \\
\hline
\end{tabular}




\begin{tabular}{|c|c|c|c|c|c|}
\hline 9 & Information & 769 & 24 & Product & 389 \\
\hline 10 & Manage & 745 & 25 & Different & 367 \\
\hline 11 & Govern & 721 & 26 & Study & 348 \\
\hline 12 & System & 698 & 27 & Need & 278 \\
\hline 13 & Platform & 667 & 28 & Provide & 256 \\
\hline 14 & Company & 657 & 29 & Work & 221 \\
\hline 15 & Knowledge & 642 & 30 & Process & 201 \\
\hline
\end{tabular}

In table 1, Technology, Data, Ecosystem, Develop words have the highest frequency. It can be seen that big data ecosystem and platform ecosystem are highly concerned at present. By summarizing and combing feature literature[16]-[17], the following topics can be obtained: (1). The development and innovation of platforms are shifting competition among enterprises to competition among platform-centric ecosystems. (2). The interaction between the platform ecosystem and the environment as well as between each ecosystem affects the utilization of market resources. The current research focuses on the design of the platform ecosystem management system.

\subsection{The topics of open ecological characteristics of cloud ERP system}

LDA method is used to analyze the literature collection in the field of open ecological characteristics of cloud ERP system, and the results are shown in Table 2.

Table 2. Most relevant term frequencies for the open and ecological characteristics of cloud ERP system.

\begin{tabular}{cccccc}
\hline$\#$ & Term & Frequency & $\#$ & Term & Frequency \\
\hline 1 & Cloud & 1443 & 16 & Process & 652 \\
2 & ERP & 1201 & 17 & Implement & 601 \\
3 & Information & 1025 & 18 & System & 598 \\
4 & Data & 977 & 19 & Adopt & 576 \\
5 & Security & 945 & 20 & Research & 541 \\
6 & Application & 920 & 21 & Quality & 487 \\
7 & Risk & 899 & 22 & Technology & 432 \\
8 & Service & 875 & 23 & Digit & 387 \\
9 & Manage & 846 & 24 & Content & 352 \\
10 & Govern & 832 & 25 & Behavior & 321 \\
11 & Model & 820 & 26 & Space & 298 \\
12 & Computing & 798 & 27 & Activity & 274 \\
13 & Business & 765 & 28 & Work & 251 \\
14 & Use & 742 & 29 & Control & 229 \\
15 & Enterprise & 731 & 30 & Software & 201 \\
\hline
\end{tabular}

In table 2, ERP, Data, Risk, Security, Application, Information words have the highest frequency. It can be seen that scholars pay more attention to the data security of cloud ERP system. By summarizing and combing feature literature[18]-[19], the following topics can be obtained: (1).Cloud ERP is an open service platform, and developers should adapt to a wider range of users with a more agile development cycle. (2).The cloud ERP system and users together constitute the cloud ecosystem, in which the data are huge and complex and users face data risks. 3.The information system in the cloud ERP system is the core of cloud services, realizing the integration of information. Distinguishing the information quality dimensions in the information management process can provide a deeper understanding of end-user satisfaction and the outcome performance of the workspace. 


\section{Construction of cloud ERP ecosystem security evaluation system}

Based on the analysis results of LDA and the concept of cloud ERP ecosystem, this paper constructs the cloud ERP ecosystem security evaluation system by expert interview.

The scientific and reasonable construction of the index system needs to go through the solicitation of expert opinions. This paper adopts the method of expert interview to conduct the first round of expert opinion survey. By investigating and sorting out the opinions of a research expert in the field of information security, a research expert in the field of cloud ERP and a research expert in the field of platform ecosystem, the evaluation index system initially established was modified and improved, and the index system was finally determined as shown in Table 3 .

Table 3. Security evaluation index system of cloud ERP ecosystem

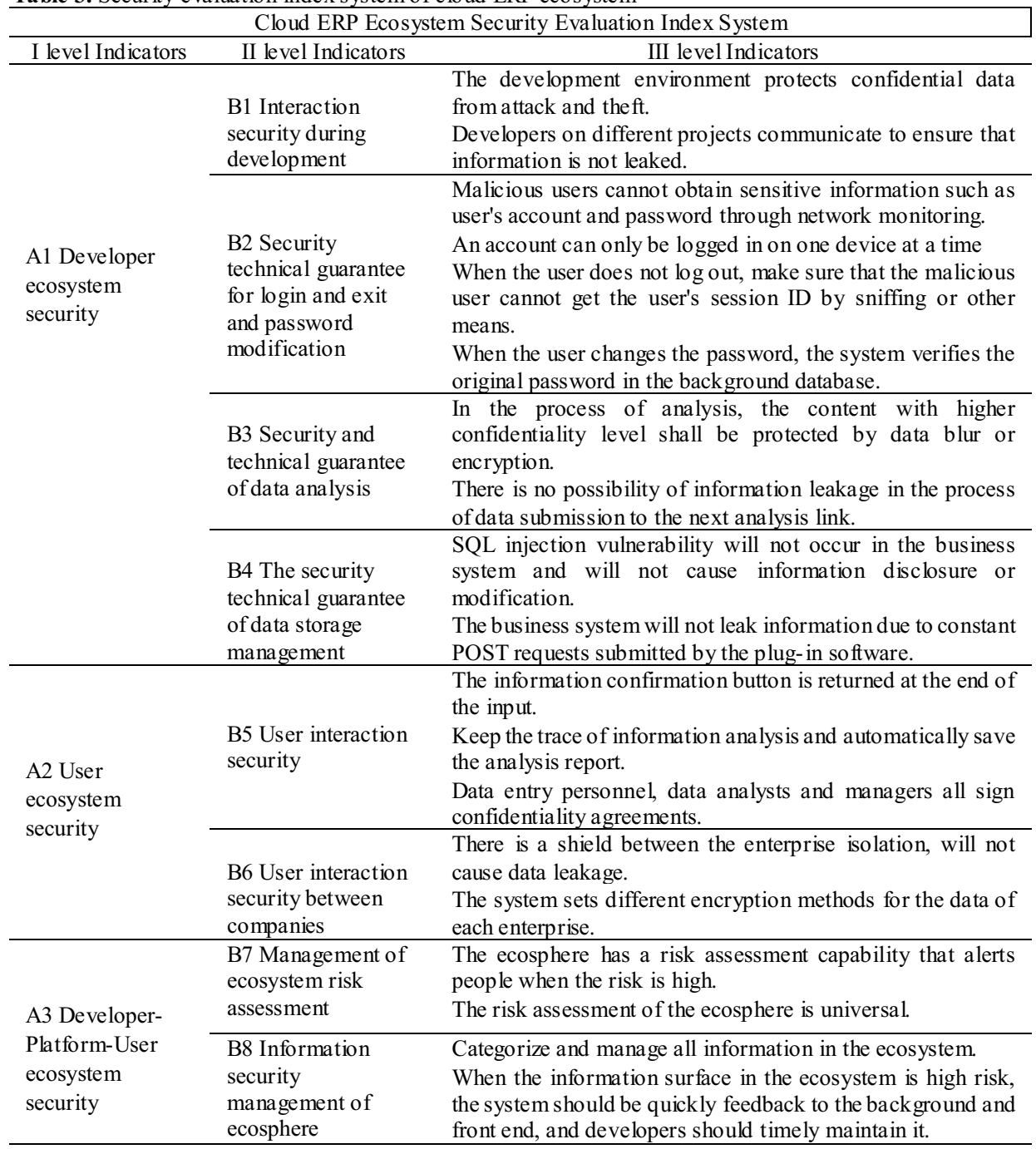

In the security evaluation index system of cloud ERP ecosystem, equal weight is adopted[20] to give weight to each three-level index. In other words, each three-level index is given the same weight, the one that meets the three-level index conditions is 1 , 
and the one that does not is zero. The number of each cloud ERP ecosystem satisfying the three-level index was comprehensively calculated, that is, the security degree of the ecosystem.

\section{Conclusion}

Cloud ERP system is more convenient and flexible than traditional ERP system. At the same time, it provides enterprises with a modern enterprise management mode with less input cost, but can meet all kinds of requirements at any time, which is the general trend of ERP development in the future under the big data environment. The ecological and open features of cloud ERP increase system interaction and bring information security risks to enterprises, and their information security threats are of great concern to enterprises. Based on the research in the fields of big data, commerce and e-commerce, this paper proposes the cloud ERP ecosystem, and analyzes the influence mechanism of the ecological openness of the cloud ERP system on the information security of the system. In this paper, the evaluation index is objectively extracted from the text database by text analysis, and the security evaluation index system of cloud ERP ecosystem is finally constructed by expert review, and the security of cloud ERP system is standardized from the ecological open perspective of cloud ERP ecosystem, so as to provide guarantee for the efficient and safe development of cloud ERP.

On the theoretical level, this research adopts the text mining based on the ecological opening characteristics of ecosphere, security and cloud ERP. On the one hand, it points out the significance of solving the cloud ERP security problem for the development of cloud ERP, and demonstrates the applicability of the concept of cloud ERP ecosystem to the research of cloud ERP security problem. On the other hand, text mining method is used to provide objective basis for the establishment of cloud ERP ecosystem security evaluation system. On the basis of practice, the concept of cloud ERP ecosystem proposed in this paper emphasizes the characteristics of cloud ERP system's ecological openness. The interaction and feedback information among developers, systems and users in the ecosystem is an important basis for cloud ERP service providers to optimize and improve system functions. In addition, the cloud ERP ecosystem security evaluation system provides reference for the information security management practice of the cloud ERP platform. Through the evaluation system, cloud ERP service providers can measure the security level of cloud ERP platform more efficiently and conveniently, and put forward relevant measures and suggestions to improve their own cloud service security management level according to the evaluation results.

\section{Acknowledgement}

The work was funded by the National Key $\mathrm{R}$ \&D Program of China (No.2019YFB1704103).

\section{References}

[1] Zhu Zonggan, Jia Yiwu. Exploratory Research on influencing Factors of Cloud ERP Service Model Classification [J]. Journal of management,2016,13(8):233-1240. 
[2] GUPTA S,MISRA S C,SINGH A, et al. Identification of challenges and their ranking in the implementation of cloud ERP : a comparative study for SMEs and large organizations[J]. International Journal of Quality \& Reliability Management,2017,34(7):1056 -1072.

[3] Erdal Cayircil, Alexandr Garaga, et al. A risk assessment model for selecting cloud service providers [J]. Journal of Cloud Computing: Advances, Systems and Applications, 2016, (5),14.

[4] Chandramohan.D, Vengattaraman.T, Rajaguru.D, Baskaran.R, and Dhavachelvan.P, "Hybrid Authentication Technique to Preserve User Privacy and Protection as an End Point Lock for the Cloud Service Digital Information".[J].IEEE-International Conference on Green High Performance Computing,2013,(40):1-4.

[5] LEWANDOWSKI J,SALAKO A O, GRACIAPEREZ A. SaaS enterprise resource planning systems: challenges of their adoption in SMEs[C] / IEEE 10th International Conference on e - Business Engineering. Coventry:IEEE,2013:56-61.

[6] Lyu Yibo, Han Shaojie, Su Jingqin and Wang Shujuan. Research on the Construction of Universitydriven Open Innovation Ecosystem[J].Management Review,2017,29(4):68-82.

[7] Tong Yuqing, Yang Yaojun .Research on Operation Mode of Ali Business Ecosystem and Platform [J].Science and Technology Management Research,2019,11:254-260.

[8] Qian Xiaocong.Study on Big Data Industry Ecology Atlas[J]. Informatization Research ,2013,39(6):4952.

[9] Shan Xue.Research on design and Evaluation of cloud Service Security Management Framework for small and medium-sized financial enterprises[D].Hefei: Hefei University of Technology, 2018.

[10] Xuexiu Chen, Chi Chen, Yuan Tao, et al. A Cloud Security Assessment System Based on Classifying and Grading[J]. IEEE Cloud Computing, 2015,2(2):58-67.

[11] Traitler H, Watzke H.J, Saguy I.S. Reinventing R\&D in an Open Innovation Ecosystem[J]. Journal of Food Science,2011,76(2):62-68.

[12] YU Yao. A Research on Construction Logic and Synergistic Mechanism of E-commerce Ecosystem in the Era of Big Data[J]. Journal of North University of Water Resources and Electric Power (Social Science Edition),2016,32(4):35-37.

[13] Tong Yuqing, Yang Yaojun. Research on Operation Mode of Ali Business Ecosystem and Platform [J].Science and Technology Management Research,2019,11:254-260.

[14] Qian Xiaocong. Study on Big Data Industry Ecology Atlas[J]. Informatization Research ,2013,39(6):4952.

[15] Sérgio Moro, Paulo Cortez, Paulo Rita. Business intelligence in banking: A literature analysis from 2002 to 2013 using text mining and latent Dirichlet allocation [J]. Expert Systems with Applications, 2015,42:1314-1324.

[16] Amrit Tiwana, et al. Platform Evolution: Coevolution of Platform Architecture, Governance, and Environmental Dynamics[J] Information Systems Research,2010,21(4):675-687.

[17] Sahar Mohammed,Osman Hegazy,Ehab E. Hassanein. A Novel Approach for Handling Security in Cloud Computing Services[J]. International Journal of Computer Applications,2013,69(5):9-14.

[18] Shivam Gupta, Subhas C Misra. Compliance, network, security and the people related factors in cloud ERP implementation [J]. International Journal of communication systems,2016,29:1395-1419.

[19] Sven Laumer,Christian Maier, Tim Weitzel. Information quality, user satisfaction, and the manifestation of workarounds: a qualitative and quantitative study of enterprise content management system users [J]. European Journal of Information Systems, 2017,26:333-360.

[20] Zhao Jin- xu, Zheng Yue-ping. Research on privacy Protection of E-government in China -- Based on 70 Investigation on "Privacy Statement" of government websites in large and medium-sized cities [J]. Egovernment,2016(7):81-93. 